\title{
LA CERÁMICA ISLÁMICA A MANO Y/O TORNETA DE EL CASTELLAR (ALCOI, ALICANTE)
}

\author{
ISLAMIC CERAMIC HANDMADE AND/OR MADE WITH A SLOW POTTER'S WHEEL IN THE CASTELLAR \\ (ALCOI, ALICANTE)
}

GERMÁN PÉREZ BOTÍ

\section{INTRODUCCIÓN}

Con motivo de la celebración del seminario «De Hispania a al-Andalus. Dinámica de cambio cultural a la luz de la Arqueología», queremos presentar el siguiente trabajo, cuyo objetivo principal es dar a conocer parte del registro material de El Castellar d'Alcoi (Alicante) y mostrar una visión más amplia de sus nuevas formas cerámicas a mano y/o torneta; así como abrir nuevas líneas de investigación sobre el tránsito del mundo tardorromano y el origen poblacional islámico en la comarca de L'Alcoià.

\section{SITUACIÓN}

Al oeste de Alcoi (Alicante) y saliendo por la carretera en dirección a Bañeres de Mariola, se aprecia a su derecha una elevación escarpada a una altura sobre el nivel del mar de 886 m conocida como El Castellar; cuyas coordenadas U.T.M son 30SYH172856, Hoja $\mathrm{n}^{\mathrm{o}} 821$ (Alcoi). Se trata de un cerro de pronunciada pendiente, protegido en sus extremos norte y este por dos grandes alineaciones naturales de rocas que dejan en su interior una especie de gran rampa en la que se sitúa el asentamiento (Fig.1).

El lugar se conoce desde hace tiempo por parte de los primeros arqueólogos alcoyanos (Vicedo, 192022; Faus et alii, 1987). A finales de los sesenta (1967 y 1969), J. Faus Cardona realizó excavaciones en diferentes zonas del yacimiento; y los materiales hallados fueron depositados en el Museo Arqueológico Municipal de Alcoi Camilo Visedo (Fig.2).

En 1973, E. Cortell y J. M. Segura elaboraron un levantamiento topográfico de las áreas y estructuras murarias excavadas por J. Faus. Un año más tarde, un equipo dirigido por A. Bazzana prospectaría la zona y realizaría dos pequeños sondeos encaminados a preparar futuros trabajos en el lugar, que a día de hoy esperan.

Las excavaciones de J. Faus dieron a conocer dos partes bastantes claras. Una zona denominada «la cima», ocupada por una construcción trapezoidal formada por varias unidades habitacionales a cada lado de un posible patio (Azuar, 1989, 134), en cuyo interior se localiza una cisterna central, que según J. M. Segura y J. Torró (1984) podría responder a una celoquia. $\mathrm{Y}$ en la segunda zona, que se extiende a lo largo de la pendiente, se localizaron una serie de viviendas rectangulares que J. Faus llamó «casas», adosadas a una posible «muralla»y dispuestas en paralelo formando terrazas, junto a otra cisterna y que las enumeró del 1 al 7 (Fig. 3). De las áreas excavadas, sólo se conocen los niveles estratigráficos en las «casas» 6 y 7. J. Faus denominó nivel A para el más superficial, nivel B el intermedio, y nivel $\mathrm{C}$ al que se encontraba en contacto con la roca madre. Estos tres niveles se establecieron en la «casa 7», y los dos primeros en la «casa 6».

Desde las primeras excavaciones, El Castellar d'Alcoi ha sido un importante referente en obras de síntesis en la arqueología islámica del País Valencià (Bazzana, 1992) o sobre el territorio de la antigua Cora de Tudmir (Gutiérrez, 1996). El investigador alcoyano J. Torró (1984) fue el primero en proporcionar una relación de éstos, indicando la existencia de cerámicas

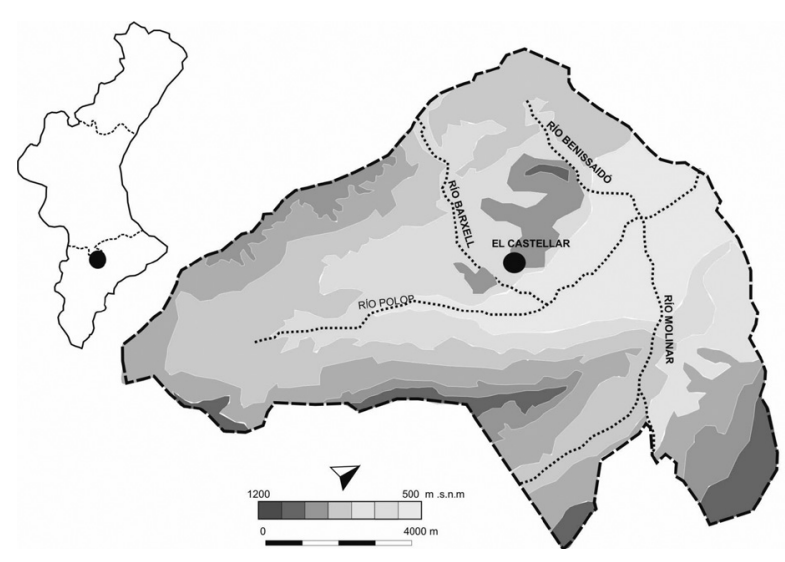

Figura 1: Situación del yacimiento de El Castellar de Alcoi (Alicante). 


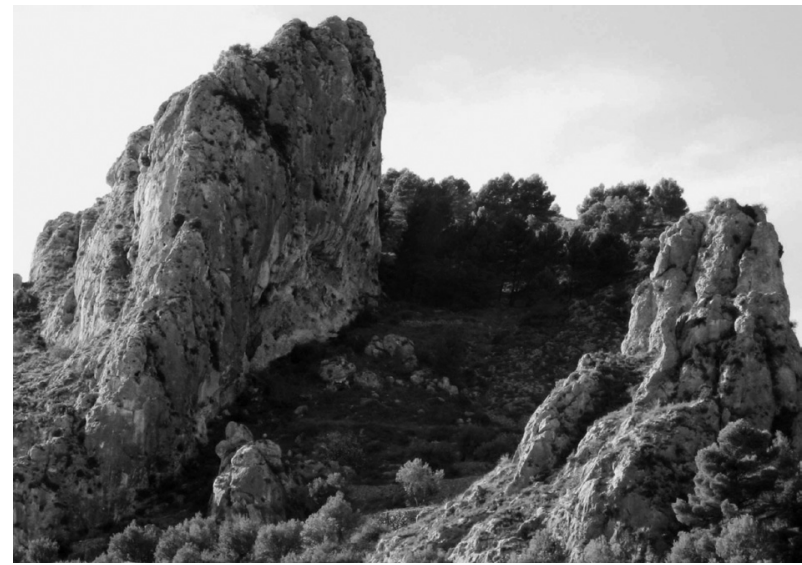

Figura 2: Vista panorámica de El Castellar de Alcoi.

para todo el período islámico. Posteriormente se realizó un estudio más pormenorizado por parte de R. Azuar (1989, 134-162), mostrando la diversidad tipológica y los numerosos motivos decorativos de los materiales cerámicos, así como los objetos realizados en hierro, bronce y hueso depositados solamente en las vitrinas del Museo. Los resultados de este trabajo permitieron ordenar de una forma más coherente la larga cronología del yacimiento en base a los materiales estudiados.

R. Azuar en su trabajo planteó la dificultad de un detenido estudio sobre los materiales de El Castellar ya que su registro era muy numeroso; y además, señaló que no había profundizado en su investigación. Partiendo de este referente, nos hemos propuesto retomar el estudio del olvidado y desconocido, y a la vez, tan nombrado en los estudios de arqueología islámica peninsular.

\section{UNA PROPUESTA DE CLASIFICACIÓN}

La larga ocupación islámica del asentamiento dificulta aplicar una clasificación concreta. Por lo tanto, se ha tenido que realizar una sistematización abierta y adaptada al registro cerámico de El Castellar. Nuestra clasificación sigue la seriación formal y funcional genérica establecida en su tiempo por G. Rosselló (1978), así como la propuesta de S. Gutiérrez (1996) para los primeros siglos de ocupación islámica. Todas las formas del registro presentan el nombre del yacimiento (Forma Castellar) junto a tres dígitos alfanuméricos. La primera variable incluye al igual que propone S. Gutiérrez (1996) aquellas piezas elaboradas a mano en el sentido estricto como las realizadas con una torneta o torno bajo $(M)$, fruto del presente trabajo. El siguiente dígito pertenece a la serie morfofuncional propuesta en su tiempo por G. Rosselló (1978). Y el tercero indica la forma en cuestión. En algunos casos puede aparecer un cuarto dígito. Éste determina alguna variable de la forma como propuso para su estudio R. Azuar (1989).

\section{EL ESTUDIO CERÁMICO}

Las formas del registro que a continuación presentamos proceden de las excavaciones realizadas por $\mathrm{J}$. Faus a finales de los años sesenta. Todas ellas se documentan en las «casas» 6 y 7 , sobretodo en esta última y en el nivel C; excepto la serie Jarrito, de la cual desconocemos su procedencia.

I.- Cazuela: entre las cazuelas modeladas a mano y/o torneta se constata una gran diversidad formal que presentamos en un trabajo anterior (Pérez Botí, 2006a). Se han registrado hasta ocho formas. Todos los ejemplares se han hallado en la «casa $7 »$ y se reparten entre los tres niveles (A, B y C). En general, las piezas se caracterizan por tener cuerpos de tendencia bitroncocónica (Castellar M5.1), semiesféricos (Castellar M5.2, Castellar M5.4; Castellar M5.8) o esféricos (Castellar M5.3, Castellar M5.5. También suelen presentar lengüetas en la parte superior del cuerpo (Castellar M5.3, Castellar M5.3.1) y asas de cinta de sección ovalada que arrancan desde el labio hasta la mitad del cuerpo (Castellar M5.6, Castellar M5.7.1). Las pastas predominantes se caracterizan por ser bastas, y con coloraciones que oscilan entre el anaranjado y el marrón oscuro. La mayoría presentan en sus superficies exteriores señales de fuego y un diámetro de la boca que gira en torno a los 17-20 cm (Fig. 4).

Entre los ejemplares de cazuela documentados cabe destacar por su cuerpo la forma Castellar M5.5, que recuerda a la serie M4 de S. Gutiérrez (1996, 77,

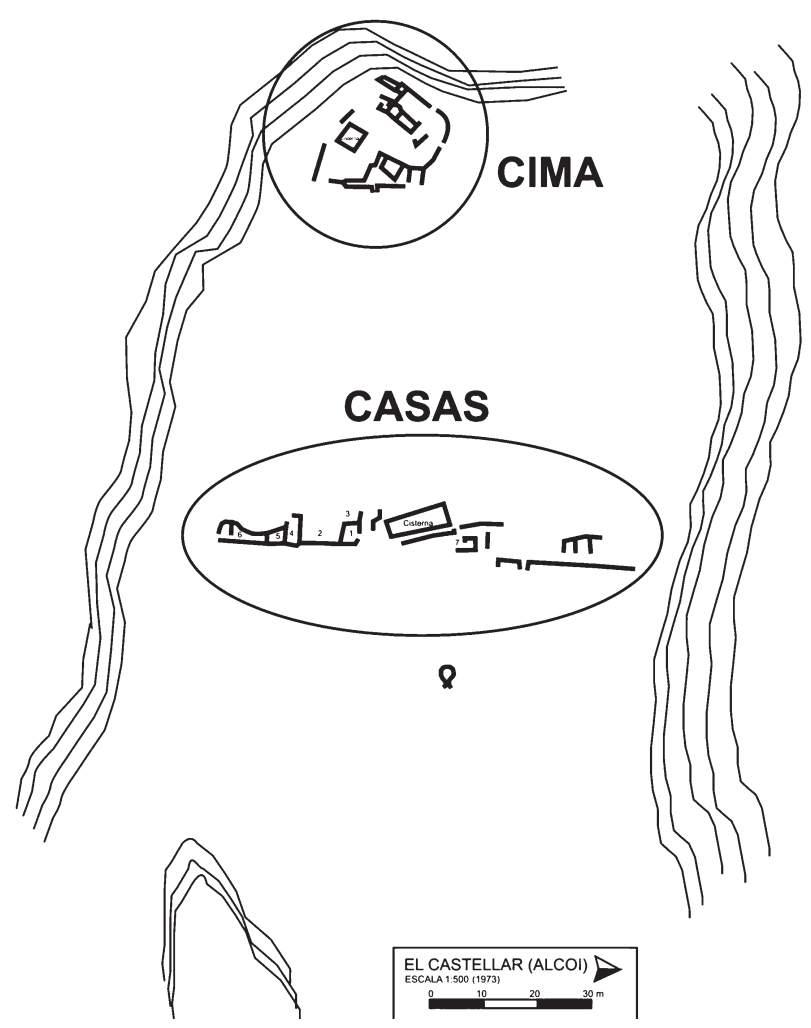

Figura 3: Planimetría de El Castellar (según Segura y Cortell, 1973). 

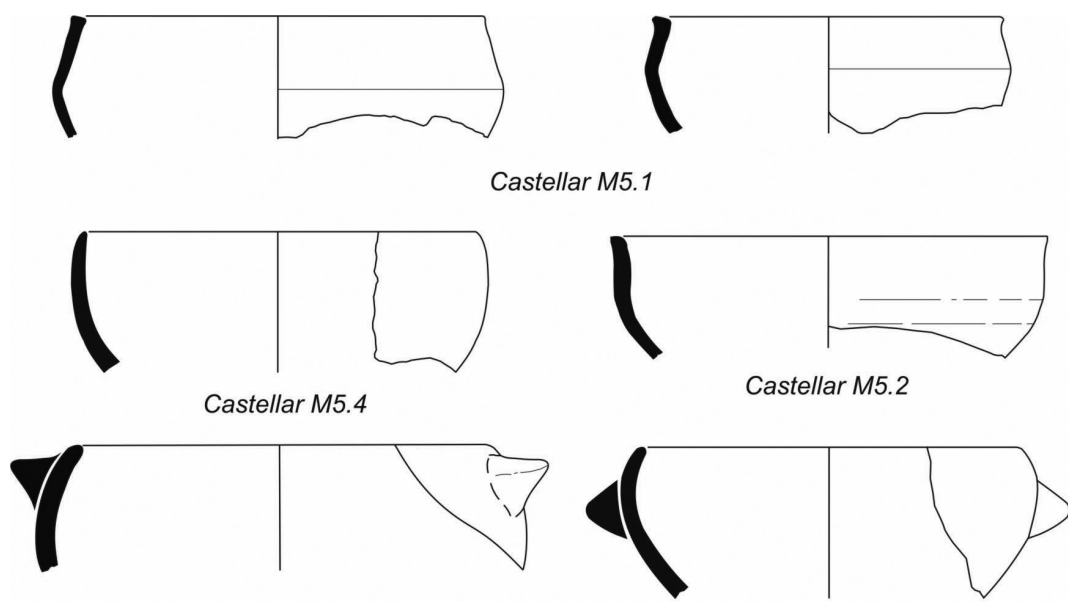

Castellar M5.3

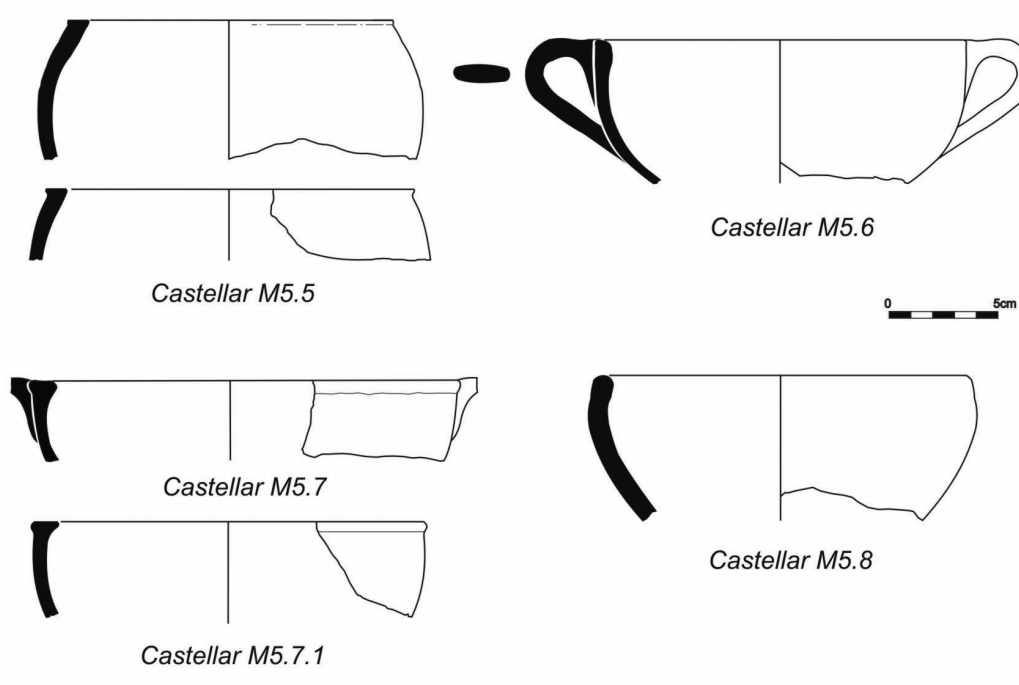

Figura 4: Serie cazuelas.

fig. 17), pero nuestro ejemplar presenta menos altura y labio plano. La forma Castellar M5.6 se asemeja al tipo I de Madinat al-Zahra, aunque aquella presenta las paredes más rectas (Vallejo y Escudero, 1999, 138); según ambos autores se enmarcan en el siglo IX y X. Si nos alejamos del área peninsular, hallamos en Nakur (Marruecos, cazuelas formalmente parecidas, con una cronología de los siglos IX-X (Acién, Cressier y Picon, 1999, 64 y 67).

2.- Marmitas: se han documentado hasta tres formas diferentes. Las dos primeras se hallaron en el nivel $7 \mathrm{C}$, mientras la última forma se desconoce. La marmita Castellar M11.1 se caracteriza por poseer una base plana y cuerpo de tendencia cilíndrica con las paredes que convergen ligeramente para formar la boca. Generalmente, suelen presentar unas lengüetas laterales pequeñas, pero también de grandes dimensiones. La pasta es basta de color marrón, con desgrasante mineral grande, y con señales de fuego en la base y la superficie inferior.

Corresponde con la forma M4.1.2 definida por S. Gutiérrez (1996, 76 y 77), presente en varios yacimientos del área de Tudmir como El Zambo (Novelda; el Forat (Crevillente); el Cabezo de las Fuentes (Albatera); Castillo de Orihuela; Tolmo de Minateda (Hellín) (Gutiérrez, 1996, 77) y Lorca (Alfar de la c/ Rojo) (Martínez Rodríguez, 1993, fig. 5). Según esta misma autora, estas marmitas características del sureste peninsular, se elaboran entre mediados del siglo VIII y mediados del IX, aunque con posibles perduraciones en contextos del siglo $\mathrm{X}$ o de principios del siglo XI (Gutiérrez, 1996; 1999, 77).

La marmita Castellar M11.3 presenta un cuerpo de tendencia esférica con cuello corto cilíndrico y borde recto de sección engrosado al exterior. A la altura del hombro aparece una banda ondulada impresa peinada. Mientras la forma Castellar M11.4 se caracteriza por un cuerpo de tendencia cilíndrica, presenta una inflexión en el hombro; cuello corto, borde exvasado ligeramente engrosado hacia el exterior. Destaca la presencia de asas de sección ovalada o alentejada, que arrancan desde el labio hasta el hombro; y al igual que la anterior, aparece una banda ondulada impresa peinada. La pasta de estas dos marmitas es compacta de 


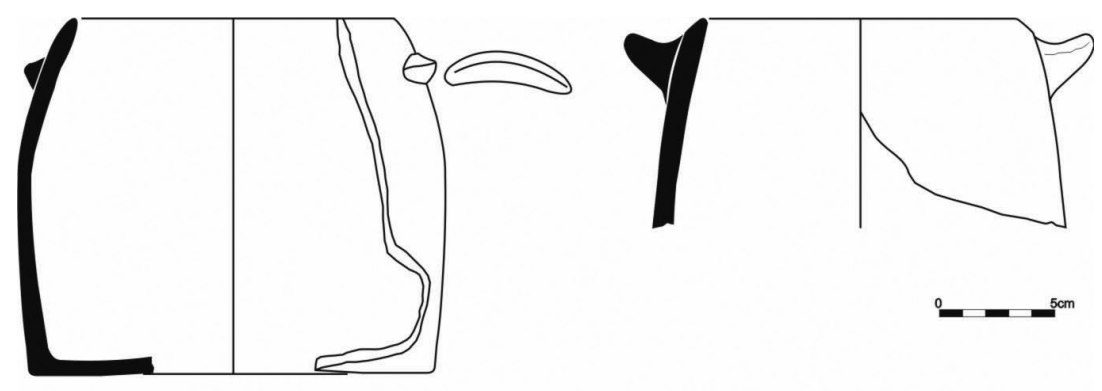

Castellar M11.1

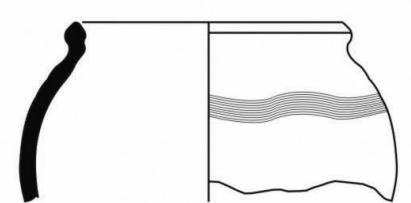

Castellar M11.3

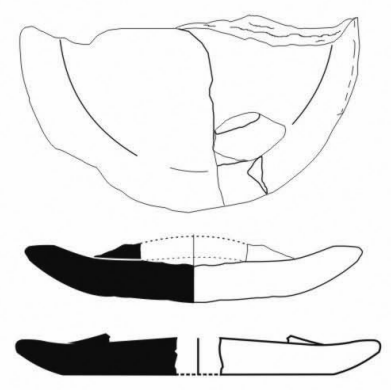

Castellar M8.1

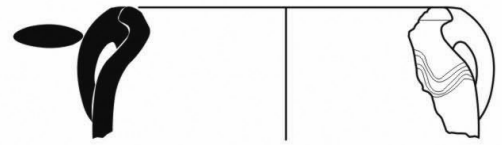

Castellar M11.4
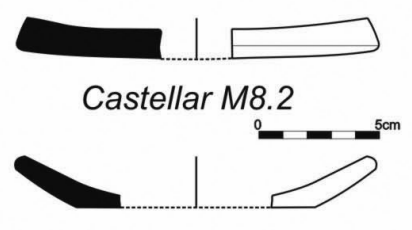

Castellar M8.3

Figura 5: Series marmitas y tapaderas.

color marrón y gris, con desgrasante mineral mediano de color negro. Y presenta señales de fuego en su superficie (Fig. 5).

Estas dos marmitas recuerdan formalmente a la M5.2 y la M5.3 de S. Gutiérrez (1996, 79. fig. 18). Éstas aparecen en contextos de la segunda mitad del siglo X y principios del XI, y se distribuyen entre Elche y la Vega Baja del Segura, hasta Murcia, como son los yacimientos de Traspalacio (Elche, Alicante); Cabezo Soler (Rojales, Alicante); La Rábita de Guardamar (Alicante) y el alfar de San Nicolás (Murcia).

3.- Tapaderas: Se han registrado seis formas de esta serie halladas en el nivel 7C, de las que sólo presentaremos las tres primeras, ya que las restantes formalmente corresponden a momentos más recientes del yacimiento. La forma Castellar M8.1 corresponde con la M30 definida por S. Gutiérrez (1996, 95, fig. 29). Son tapaderas de pequeño tamaño y base plana o ligeramente convexa, de forma redonda y con un asa de cinta central. La forma Castellar M8.2 se caracteriza por poseer un labio biselado al exterior, y recuerda a la M30.1.1 de S. Gutiérrez (1996, 95, fig. 29). Las tres formas están elaboradas con una pasta basta de color marrón y gris, con desgrasante mineral grande; y también presenta señales de fuego en toda su superficie (Fig. 5).

Estas tapaderas se adscriben a contextos del siglo $\mathrm{X}$ o principios del siglo XI, y se documentan en varios yacimientos del área de Tudmir como el Tolmo de Minateda (Hellín); El Zambo (Novelda) o la Rábita de Guardamar. Mientras que la forma Castellar M8.3 se caracteriza por presentar el borde distal convexo. Posiblemente estemos ante una producción local, al igual que las dos anteriores.

4.- Jarritos: sólo se ha constatado una forma Castellar M4.1, de la cual se desconoce su procedencia estratigráfica. Es un recipiente de pequeño tamaño; cuerpo de tendencia ovoide, cuello cilíndrico, y borde recto ligeramente exvasado. Presenta un arranque de asa de cinta vertical en la parte inferior del cuerpo. Aparecen en su superficie dos filetes gruesos verticales pintados en óxido de hierro. La pasta es compacta de color anaranjado, con desgrasante mineral grande. 

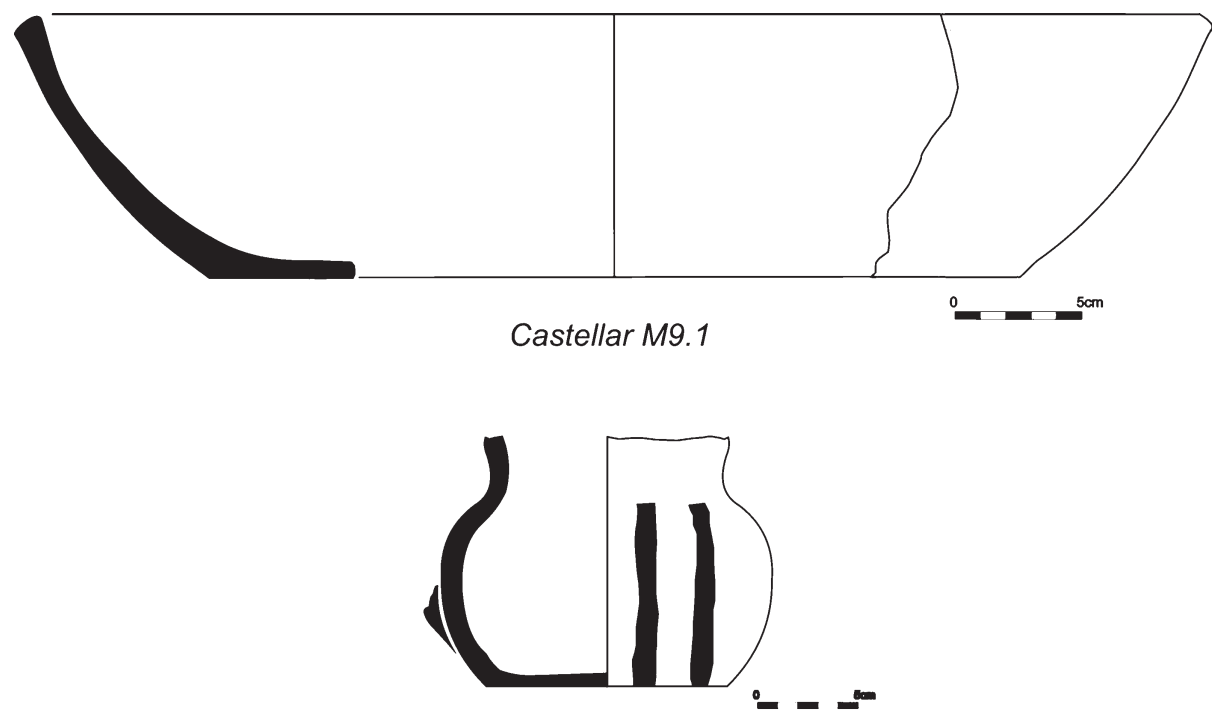

Castellar M4.1

Figura 6: Series alcadafe y jarrito.

En estos momentos de la investigación desconocemos piezas similares (Fig. 6).

5.- Tinajas: La serie Tinaja es de las que menor registro aporta el yacimiento. Destaca una sólo pieza realizada a mano, la forma Castellar M15.1. Se trata de un recipiente de gran tamaño y boca amplia. Cuello de tendencia cilíndrica y borde recto con sección engrosada curvo al exterior, y labio plano. Presenta una decoración impresa a modo de motivos circulares a lo largo de la superficie del labio. La pasta es basta de color marrón, con desgrasante mineral grande. Desconocemos piezas similares en el ámbito regional en el que se sitúa el asentamiento. Posiblemente nos encontremos ante una producción de carácter local (Fig. 7).

6.-Ataifores: sólo se ha registrado una forma (Castellar M1.1), hallada en el nivel 7C. Se trata de un recipiente bajo de boca amplia; base plana y cuerpo de tendencia troncocónica invertida con paredes rectas y borde exvasado, y labio curvo. La pasta basta de color anaranjado, con desgrasante mineral grueso. Por sus características, recuerda a la forma M27.4.1 de S. Gutiérrez (1996, 92, fig. 26), aunque ésta presenta una pared ligeramente curva. Estas piezas se localizan en la cora de Tudmir, como es el caso de la Rábita de Guardamar (Gutiérrez 1996, 93); calle San Nicolás (Murcia) (Gallego Gallardo, 1993, 126, lám. 9). Fuera de esta zona, las encontramos en la ciudad de Valencia (Blasco et alii, 1987, fig. 4,3) o en El Maurete (Motril, Granada) (Gómez Becerra, 1992, 48 y 49). Para el área de Tudmir se propone una cronología de finales del siglo IX y siglo X (Gutiérrez, 1996, 93 (Fig. 7).

7.- Alcadafe: Al igual que la serie anterior se ha documentado una sola pieza en el nivel 7A. La forma Castellar M9.1 corresponde a un recipiente bajo de gran tamaño y boca muy amplia; base plana, paredes exvasadas; cuerpo troncocónico invertido y borde exvasado con sección engrosado curvo exterior y labio curvo. Recuerda a la forma M28.1 de S. Gutiérrez (1996, 93 y 94, fig. 27) hallada en el alfar de San Nicolás (Murcia) (Gallego Gallardo, 1993, lám. 9). Respecto a su cronología, se plantea una adscripción indeterminada ya que se documentan por todo el al-Andalus y a lo largo de su historia, aunque no podemos descartar un posible origen en el siglo X (Fig. 6).

8.- Tannūr: el estado de esta serie es bastante fragmentario, aunque la mayoría de las piezas documentadas presentan las mismas generalidades. Éstas proceden de los niveles $6 \mathrm{~B}$ y $7 \mathrm{C}$. La forma característica es la Castellar M14.1. Consiste en un útil abierto por ambos lados de gran tamaño, con paredes rectas y cuerpo de tendencia troncocónica, y borde engrosado de labio plano. La pasta es basta de color marrón, con un desgrasante mineral muy grueso y con señales de fuego por toda su superficie. En la exterior, aparecen unos cordones plásticos con impresiones digitales en el inicio de la boca o en su parte inferior. Y en el interior, profundas líneas incisas peinadas (Fig. 7).

Corresponde a la forma M9.2 de S. Gutiérrez (1996, 86 y 87). Según esta autora, esta forma sólo se documenta en los yacimientos del área de Tudmir, como la Rábita de Guardamar; el Zambo (Novelda); ¿Tolmo de Minateda? (Hellín), Castillo de la Sierra (Callosa de Segura, Alicante); Cabezo Soler (Rojales, Alicante); ¿Cabezo del Moro? (Barinas, Murcia); Albacete; El Sompo (Cocentaina, Alicante), de donde procede una pieza completa (Gutiérrez, 1990-91, fig. 2.3).

Respecto a la cronología de estas piezas, se documentan a partir del siglo IX; pero posiblemente 

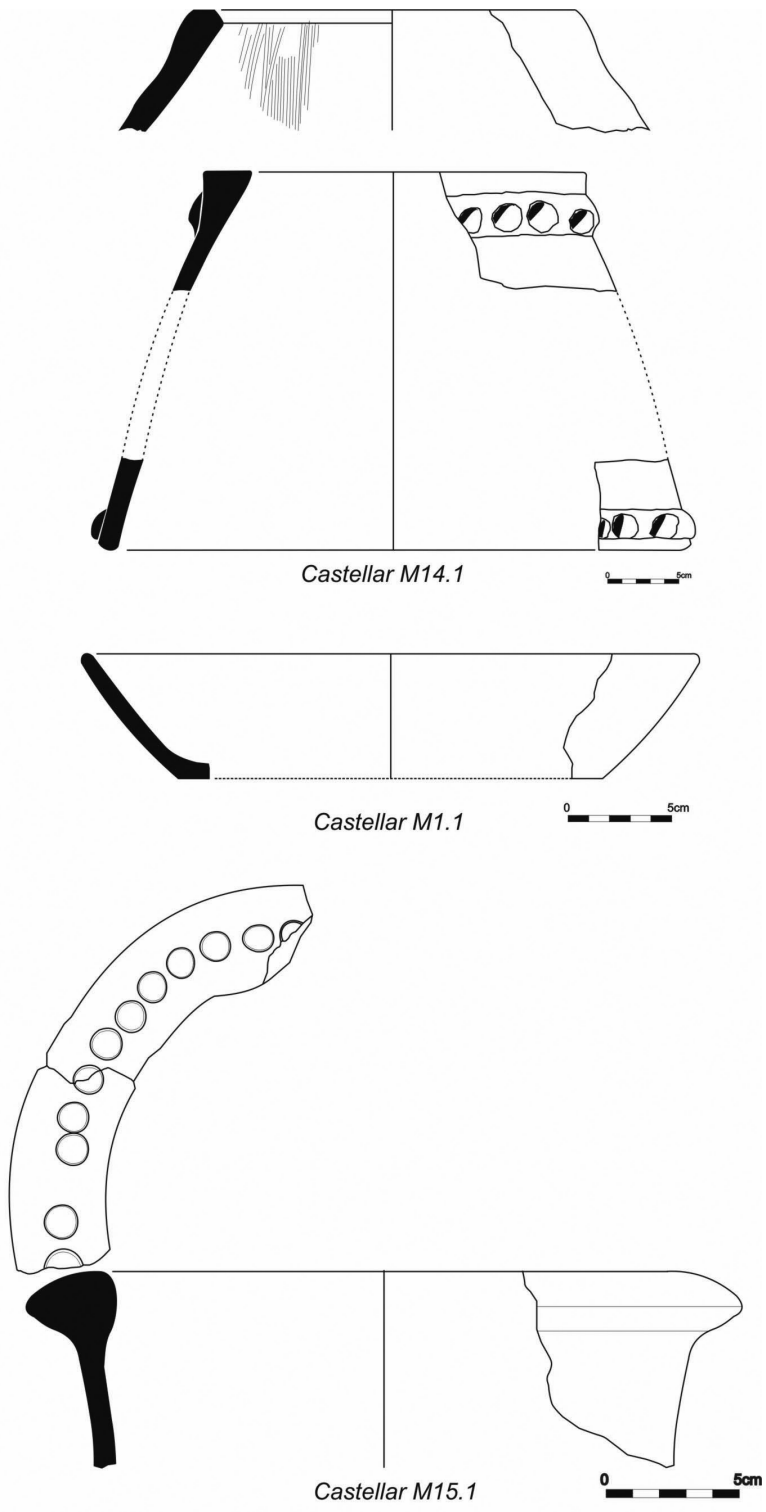

Figura 7: Series tannūr, ataifor y tinaja.

nuestras piezas corresponden a los siglos X y XI, aunque pueden perdurar durante los siglos XII y XIII (Azuar, 1989, 112-117).

\section{CONCLUSIONES}

En base al registro cerámico presentado podemos retomar y plantear la siguiente pregunta: ¿Cuál es el origen de El Castellar de Alcoi en época islámica? En anteriores estudios, los investigadores consideraban dos hipótesis sobre su origen. Por una parte, R. Azuar (1989) proponía una primera ocupación correspondiente al siglo XI, en época taifal. Y por otra, autores como J. Torró $(1984 ; 1992)$ y P. Guichard (1990) situaban el inicio del asentamiento en el siglo X, en plena época califal.
Según J. Torró, en el siglo X, durante el Califato de Córdoba la población de estas tierras se encontraba totalmente islamizada, y El Castellar ya estaba claramente formado como hábitat fortificado.

Por otro lado, R. Azuar (1989) y S. Gutiérrez (1996), a partir del registro arqueológico planteaban que todos los materiales estudiados no podían ir más allá de un contexto del siglo XI. Sólo destacaba la presencia de una marmita a mano (Castellar M11.1), que se encontró sobre la propia roca madre en el nivel 7C; y publicada por J. Torró (1984) y estudiada por estos dos autores. Según S. Gutiérrez (1996) la pieza corresponde a la forma M4.1.2. Sobre su origen, estas marmitas aparecen en la segunda mitad del siglo VIII y siglo IX, aunque con posibles perduraciones en contextos del siglo X y principios del XI. Asimismo, su hallazgo aislado en un nivel claramente de principios del siglo XI (Azuar, 1989, 148), y en el contexto geográfico en el que este yacimiento se ubica, planteaba un inicio taifa del asentamiento. Además se interpretaba como «una pieza residual, procedente, casi con total seguridad, del exterior del poblado» (Gutiérrez, 1996, 366).

Partiendo de estas dos propuestas, podemos volver sobre la cuestión planteada a partir del registro. Antes de entrar en discusión, presentaremos la dificultad arqueológica del yacimiento, y de su propia investigación. Los materiales estudiados hasta estos momentos por parte de R. Azuar (1989) pertenecen solamente al repertorio de las vitrinas del Museo. Por lo tanto, como mencionaba este autor, no era un estudio pormenorizado y exhaustivo. Otro aspecto a tener en cuenta, es la propia excavación. Si leemos atentamente el diario de J. Faus, los niveles establecidos son totalmente artificiales, propios de esa época. De todas las «casas» parcialmente excavadas, sólo en la 6 y la 7 se documentan niveles. Para la casa 6, el A y B; y para la 7, el A, B y C. El nivel A es el más superficial, mientras que el $C$ está en contacto con la roca madre, estando la B en una posición intermedia. Esta artificialidad estratigráfica se corrobora en el mismo registro cerámico, ya que muchos fragmentos de una misma pieza se pueden localizar en distintos niveles. Así como en el nivel 7C, podemos encontrar fragmentos de clara adscripción almohade mezclados con otros más antiguos. Pero partiendo de un hecho evidente, como es el contacto del nivel 7C con la roca madre, junto al nuevo registro clasificado, nos abre una nueva posibilidad sobre su origen.

El único testimonio cerámico que aportaba una relativa antigüedad del asentamiento era el hallazgo aislado de la forma Castellar M11.1 en el nivel 7C. Pero a partir del presente estudio, el número de piezas elaboradas a mano y/o torneta se amplia, y sobre todo en el mismo nivel que la forma Castellar M11.1. Es el caso de aquellas marmitas con decoración peinada (Castellar M11.3); cazuelas (Castellar M5.1); ataifores (Castellar M1.1); tapaderas (Castellar M8.1; Castellar M8.2, Castellar M8.3); tinajas (Castellar M15.1) y Tannūr (Castellar M14.1). En el nivel 7B, 


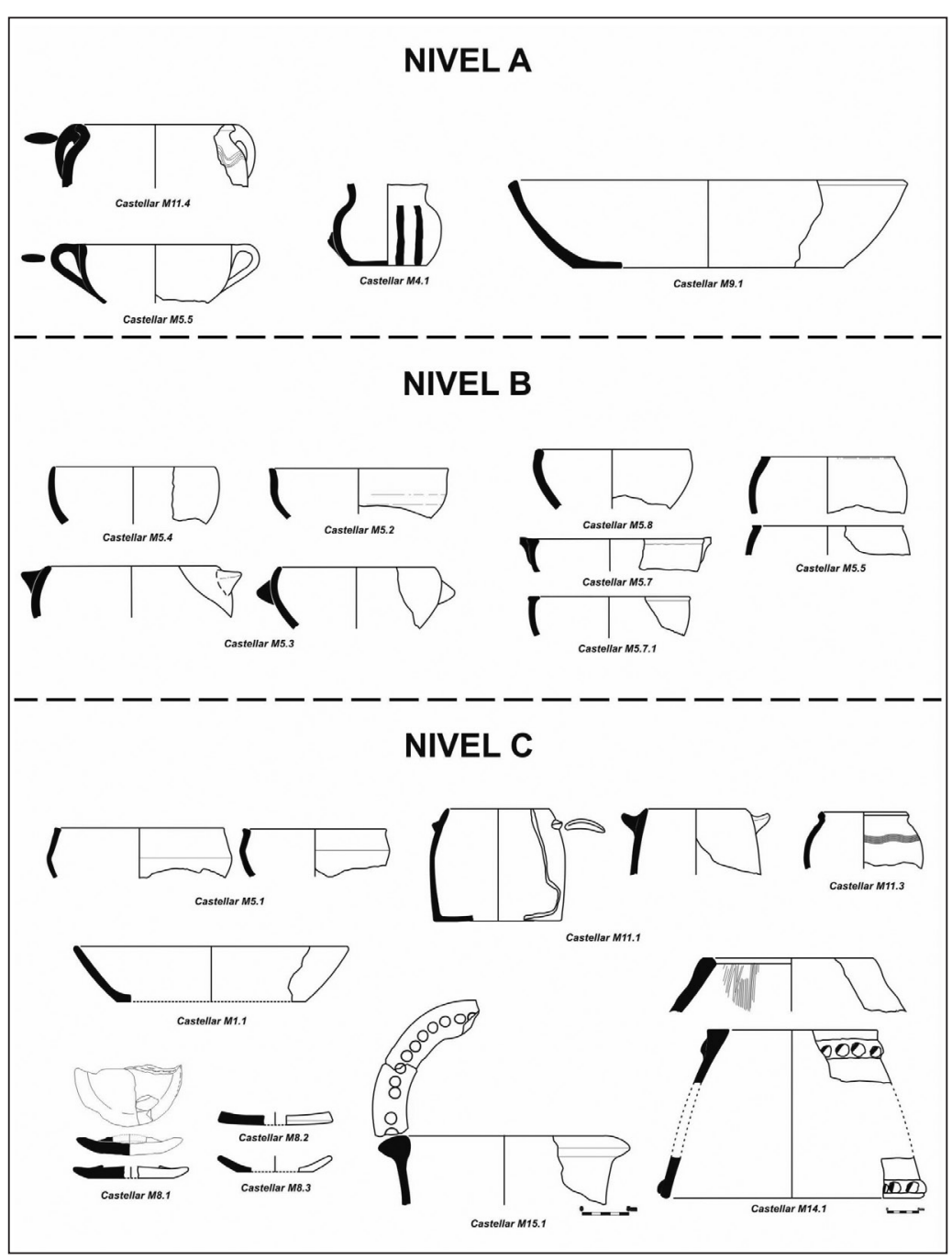

Figura 8: Propuesta estratigráfica correspondiente a la "casa" 7.

también encontramos cazuelas (Castellar M5.2; Castellar M5.3; Castellar M5.4; Castellar M5.5). Así como en el resto del yacimiento: marmitas (Castellar M11.4; y jarritos (Castellar M4.1).

Como en líneas anteriores se ha mencionado, parte de las formas halladas en el nivel 7C presentan una adscripción cronológica en torno a finales del siglo IX (Castellar M1.1; Castellar M11.1) y a lo largo de todo el siglo X (Castellar M5.5; Castellar M8.1; Castellar M11.3; Castellar M9.1), llegando otras posiblemente al XI (Fig. 8).

Otro dato a tener en cuenta, es el registro de nuevas formas en el nivel $7 \mathrm{C}$ de las que se desconoce su procedencia, ya que pensamos que son una producción local como las cazuelas (Castellar M5.1; Castellar M5.2), la tinaja (Castellar M15.1) o la tapadera (Castellar M8.3).

En conclusión, a la vista de este conjunto de cerámicas elaboradas a mano y/o torneta podemos sugerir que el Castellar de Alcoi se acerca más a un origen en torno a finales del siglo IX y principios del siglo $\mathrm{X}$ que al periodo taifa, como también corrobora el registro material realizado a torno en un anterior trabajo (Pérez Botí, 2006). Posiblemente, la mayoría de las piezas del nivel 7C fueran elaboradas en el propio yacimiento o en áreas muy cercanas. Aunque como señala J. Torró (1984), en el siglo X toda el área de Alcoi estaba totalmente islamizada, creemos que El Castellar aún no se había relacionado con los circuitos comerciales a principios de siglo. Esto nos hace pensar en una producción local autosufiente, que cambiará a finales del siglo X, y sobre todo en el siglo XI, en la que las producciones a torno se generalizarán (Fig. 9).

Toda hipótesis arqueológica necesita de una verificación estratigráfica, solamente una excavación sistemática del yacimiento permitiría corroborar, o no, nuestra propuesta planteada.

Germán Pérez Bot

C/ Roig de Corella, 3 03804 Alcoi

germangp27@hotmail.com 


\section{BIBLIOGRAFÍA}

ACIÉN, M.; CRESSIER, P. y PICON, M., 1999: «La cerámica a mano de Nakūr (ss.IX-X producción bereber medieval», Arqueología y Territorio, 6, actas del Coloquio La cerámica andalusí. 20 años de investigación, 47-69, Jaén.

AzuAR, R., 1989: Denia islámica. Arqueología y Poblamiento, Alicante.

BazzAnA, A., 1992: Maisons d'Al-Andalus. Hábitat medieval et estructures du peuplement dans l'Espagne Orientale. Madrid.

Blasco, J., CAmps, C. y MonRaval, M., 1987: «Reconstrucción de viviendas islámicas tras ser destruidas por una riada (siglo XI)», II Congreso Nacional de Arqueología Medieval (Madrid, 1987), II, 467-476, Madrid.

Faus BarberÁ, J., Aragonés, V., FAus CARdona, J. y PLA, R., 1987: Un catálogo de yacimientos de a montaña alicantina, Alcoi.

FAUS BARBERÁ, J., 1967-69: Diario de la excavación de El Castellar, Alcoi.

GALlEGO J., 1993: «Memoria de las excavaciones de urgencia realizadas en c/ San Nicolás, 6. Riquelme (Murcia. Octubre 1988/Enero 1989)», I Jornadas de Arqueología Regional, Memorias de Arqueología, 4, 351-380, Murcia.

Gómez Becerra, A., 1990: «El yacimiento de «El Maurete» (Torrenueva, Motril»»», en GÓMEZ BECERRA, A.; MalpicA, A. y Trillo, C.: De Paterna a Mutrayil (cerámica medieval), 33-37 y 53-111, Motril.

GUICHARD, P. et alii, 1990: «La cerámica con decoración 'verde y manganeso'», La cerámica islámica de la ciudad de Valencia, 71-95, Valencia.

GutiÉRreZ LlORET, S., 1990-91: «Panes, hogazas y fogones portátiles. Dos formas cerámicas destinadas a la cocción del pan en al-Andalus: el hornillo (tannūr) y el plato (tãbaq)», Lucentum, IX-X, 161-175.

GutiÉRREZ Lloret, S., 1996: La Cora de Tudmir: de la antigüedad tardia al mundo islámico. CCV, 57, Madrid-Alicante.

GUTIÉRREZ Lloret, S., 1999: «La cerámica emiral de Madinat Lyih (el Tolmo de Minateda, Hellín, Albacete)», Arqueología y Territorio, 6, Actas del Coloquio: La cerámica andalusí. 20 años de investigación, 71-111, Jaén.

MARTínez RodrígueZ, A., 1993: «Excavación de urgencia en el cerro de Peña María (Lorca)», I Jornadas de Arqueología Regional. Memorias de Arqueología, 4, 289 300, Murcia.

PÉREZ Botí, G., 2006a: «La evolución formal y cronológica de la cazuela andalusí en El Castellar (Alcoi, Alacant)», Recerques del Museu d'Alcoi, 15, 85-92, Alcoi.

PÉrez Botí, G., 2006 b (e.p.).: «El origen islámico de El Castellar (Alcoi, Alacant) a partir del registro cerámico», Congreso sobre la Historia de Alcoi, 750 años, Alcoi.

Roselló Bordoy, G., 1978: Ensayo de sistematización de la cerámica árabe de Mallorca, Palma de Mallorca.

TORRÓ ABAD, J., 1984: «Arqueología medieval de Alcoi y su entorno», Alcoy, Prehistoria y Arqueología. Cien años de investigación, 277-309, Alcoi.

TORRÓ ABAD, J., 1992: La formació d'un espai feudal. Alcoi de 1245 a 1305, Valencia.

VAllejo Triano, A. y Escudero ArandA, J., 1999: «Aportaciones para una tipología de la cerámica común califal de Madinat al-Zahra». Arqueología y Territorio, 6, Actas del Coloquio: La cerámica andalusí. 20 años de investigación, 133-176, Jaén.

VISEDO, R., 1920-22: Historia de Alcoy y su Región, Alcoi. 